\title{
SOLUCIÓN DE DISPUTAS EN LA OMC: UN ANÁLISIS A PARTIR DE LA SOCIOLOGÍA DE PIERRE BOURDIEU
}

\author{
José Augusto Fontoura Costa
}

WTO DISPUTE SETTLEMENT: A SOCIOLEGAL ANALYSIS FROM THE PIERRE BOURDIEU'S PERSPECTIVE

\section{RESUMO}

EL SISTEMA DE ARREgLO DE DISPUTAS DE LA OME ES MUY ESTUDIADO Y PRODUCE LITERATURA MUY DIVULGadA. SIN EMBARGO, HAY POCOS ESTUDIOS EMPÍRICOS SOBRE LOS PERFILES DE MIEMBROS DE GRUPOS ESPECIALES Y DEL ÓRgANo DE APELACIÓN Y, ASIMISMO, SU INFLUENCIA EN LA ARTICULACIÓN DE UN CAMPO JURÍDICO INTERNACIONAL AL DERREDOR DE LOS ÓRgANOS DE ARREGLO DE DISPUTAS. ESE ESTUDIO TIENE EL OBJETIVO HACER DISPONIBLES ALGUNAS INFORMACIONES Y ANALIZARLAS BAJO LA LUZ DE ALGUNOS CONCEPTOS DE LA Sociología de Pierre Bourdieu, en particular da de campo JURÍDICO Y DE HABITUS, PARA COMPRENDER COMO SE PRODUCE LA JURIFICACIÓN INTERNACIONAL. LOS RESULTADOS INDICAN SIMILITUDES ENTRE EL CAMPO JURÍDICO DE LA OMC Y LOS CAMPOS JURÍDICOS ESTATALES.

\section{PALAVRAS-CHAVE}

OMC; SISTEMA DE ARREGLO DE DISPUTAS; JURIFICACIÓN internacional; CAMpo Jurídico; Pierre Bourdieu; Perfiles DE TOMADORES DE DECISIONES

\begin{abstract}
THE WTO'S DISPUTE SETTLEMENT SYSTEM IS A MUCH STUDIED SUBJECT AND THE LITERATURE THEREON IS WIDESPREAD. NEVERTHELESS, THERE ARE FEW EMPIRICAL STUDIES ON PANELISTS' AND APPELLATE BODY MEMBERS' PROFILES AS WELL AS THEIR INFLUENCE ON THE ARTICULATION OF AN INTERNATIONAL LEGAL FIELD AROUND THE DISPUTE RESOLUTION ORGANIZATIONS. THIS STUDY AIMS TO MAKE AVAILABLE SOME INFORMATION ON THESE PROFILES AND ANALYZE THEM UNDER THE LIGHT OF SOME CONCEPTS OF PIERRE BOURDIEU'S SOCIOLOGY, MAINLY THE LEGAL FIELD AND THE DEVELOPMENT OF SOCIAL HABITUS, IN ORDER TO UNDERSTAND HOW INTERNATIONAL LEGALIZATION OF THE TRADE LAW TAKES PLACE. THE RESULTS POINT OUT THE SIMILARITIES BETWEEN THE WTO'S LEGAL FIELD AND THE STATE LEGAL FIELDS.

\section{KEYWORDS}

WTO; DISPUTE SETTLEMENT SYSTEM; INTERNATIONAL Legalization; Legal field; PIerRe Bourdieu; deCISIONMAKERS'PROFILES
\end{abstract}

\section{INTRODUCCIÓN}

Se hizo moneda corriente, en los estudios respeto al derecho de la Organización Mundial del Comercio (OMC), la dicotomía propuesta por John Jackson (1997), que habla del cambio histórico del sistema de solución de disputas (SSD) de un modelo de "diplomacia orientada por el poder" para una "diplomacia orientada por reglas". Aunque su utilización sea demasiado simplificadora, es traducida en el discurso sobre el SSD de la OMC como parte de un proceso natural de jurificación por el que pasan 
las instituciones y organizaciones internacionales, entendida como el incremento de la clareza, atribución de obligaciones y delegación de la aplicación a órganos no estatales (ABBOTT et al.: 2000).

Sin embargo, no es razonable aceptar tales simplificaciones, las cuales apartan de la comprensión de los cambios institucionales los aspectos sociológicos de la construcción efectiva de campos de acción, lo que es resultado de estructuras de sentido históricamente establecidas a partir de los conflictos y conciertos en la búsqueda de valorización de capitales simbólicos internacionales. Desde luego, los conceptos de la sociología de Pierre Bourdieu se muestran extremadamente útiles para tratar el tema de la jurificación internacional, aunque algunas diferencias deban de ser trazadas en contraste con la formación del campo jurídico estatal o interno. Aunque no abarque todo el Derecho Internacional, el Derecho del Comercio Internacional y, en particular, de la OMC se presenta como un caso importante e interesante para el estudio de cómo se articula un SSD internacional.

En términos formales, el SSD de la OMC se estructuró en torno a dos instancias de ejercicio de la jurisdicción: (1) los grupos especiales (panels), que son tribunales ad hoc formados para solucionar disputas y (2) el Órgano de Apelación (OA), al que la parte sucumbiente puede recorrer para intentar reverter los informes de los grupos especiales y al cual se devuelve materia de derecho, pero no de hecho. Aunque los informes de grupos especiales o del OA a penas se conviertan en decisiones vinculantes si adoptados por el Órgano de Solución de Disputas (OSD), en el cual están representados todos los Estados Miembros de la OMC y sería el responsable por el control político de los fallos, no es probable que se pueda bloquear la adopción de una decisión, puesto que sería necesario el consenso de todos los Miembros, incluso el vencedor de la disputa.

Aunque no se vaya, en ese artículo, discutir los procedimientos de elección de los componentes de grupos especiales y del OA, es importante rememorar cuales son esos. Los grupos especiales pueden ser formados de dos maneras suplementarias: (1) el Secretariado de la OMC indica los componentes y las partes en la disputa los aprueban o, si estas no llegan a un acuerdo en 20 días, (2) por el DirectorGeneral, a pedido de la parte reclamante. El OA es formado por personas indicadas por los Miembros y aprobadas en el OSC, luego de entrevistas en los Consejos y, en la práctica, un periplo por las representaciones en Ginebra que ya se ha nombrado "una especie de canje político de caballos" (CARTLAND: 2003, 214).

La propuesta de ese artículo, por lo tanto, es que se puede concentrar el análisis de la construcción de un campo jurídico de la OMC en esos dos tipos de órganosgrupos especiales y OA, para evaluar los perfiles de los componentes de grupos especiales y del OA, con la finalidad de identificar si hay indicadores de una juridificación del SSD en el sentido, distinto del de Abbott et al. (2000), de la creación efectiva de un campo jurídico internacional. Se describen acá, por lo tanto, algunas características 
de los juzgadores, sobre todo las relacionadas con la formación en Derecho y la carrera profesional.

\section{CAMPO JURÍDICO Y CAMPO JURÍDICO INTERNACIONAL}

La descripción de campo jurídico en la sociología de Pierre Bourdieu (2001b) emplea algunas categorías centrales de su análisis, como las de habitus y capital simbólico, del cual el capital cultural y social son especies, para describir la construcción de la práctica jurídica como resultado de la acción y de las tensiones en términos de posiciones relativas de los actores sociales. Como pantalla en la que se proyectan los juegos y peleas por capitales y activos se pone la cuestión de la dominación mediada por la violencia simbólica que hace posible utilizar la fuerza monopolizada por el Estado de una manera que se pone como técnicamente legítima. Por lo tanto, la crítica del legalismo como instrumento de ocultación de la arbitrariedad y domesticación de los conflictos sociales surge como coherente con la tradición marxista de la sociología como instrumento de revelación de las estructuras de dominación, aunque las dinámicas del campo se produzcan en un espacio de doble alienación: (1) en las luchas internas, el habitus de los participantes no permite la incorporación de la crítica de la dominación, sino mediante su desvirtuación como argumentos técnicos legitimados y (2) la posibilidad de manutención del campo jurídico como operacional para la dominación a través de los aparatos estatales se sostiene sobre la negación de la violencia ocultada y la afirmación de los valores de justicia y de la buena técnica, que neutraliza el carácter político de la jurisdicción.

En consecuencia, no es equivocado afirmar que la teoría del campo jurídico surge, en Bourdieu, como una explicación de la operación de los aparatos estatales por medio de la conversión de la fuerza en violencia simbólica bajo la opacidad del discurso técnico del derecho y, por lo tanto, de una tecnización de la política. Sin embargo, como no se atribuye la conciencia de esa operación a los sujetos sociales inmediatamente involucrados en la actividad cotidiana, los cuales juegan sus roles, naturalizados en habitus constitutivos de la personalidad, en mercados de activos simbólicos, esa tampoco se pone como condición previa necesaria del cambio, el cual resulta de los conflictos y luchas por centralidades y valorización de capitales. Así, la teoría de los campos sociales importa las dinámicas del mercado y, con ellas, la mano invisible qué comanda la producción y distribución de bienes.

En otras palabras, de la perspectiva del jugador no se puede mirar toda la cancha a la vez y tampoco se pueden calcular las posiciones y velocidades de todos los jugadores: como no se controlan (o conocen) todas las variables, la autocrítica es siempre incompleta, porque limitada a una perspectiva posicional de base - como resta claro de la sociología del hacer académico. Por consiguiente, la crítica no determina, 
aunque influencia reflexivamente, los habitus y las composiciones de campo, lo qué implica una teleonomía de las composiciones sociales históricas; las teleologías son siempre limitadas, ya a las descripciones ex post facto qué identifican los fines con el estado actual, ya a las utopías, en que los fines están, de modo idealista, puestos en el futuro. La percepción de una Historia en que los actores eligen sus guiones en conformidad con estrategias limitadas por percepciones de la realidad enclavadas en los habitus reconocidos se acerca más de la biología evolutiva darviniana y, por lo tanto, a otra elaboración teórica en que, como en el mercado de los economistas, los resultados reflejan la interacción de los muchos elementos involucrados, pero cuyo resultado no es previamente determinado o, por lo menos, previsible desde la limitada perspectiva de los actores.

Es importante resaltar ese rasgo de la sociología de los campos porque, cuando se busca el análisis de la formación de ámbitos internacionales de actividad jurídica, la percepción de las dinámicas posicionales y los intercambios de capital se muestran instrumentos muy adecuados para la comprensión de la realidad, mientras que la conversión de la fuerza en violencia simbólica no se pone como problemática de fundo porque no existe, en el ámbito internacional o mundial, alguna entidad análoga al Estado y, por lo tanto, las cuestiones referentes a como se construyen nuevos campo jurídicos o asemejados, si no se les puede nombrar así, en ámbitos no estatales. Más que la cuestión de la dominación por el aparato estatal están puestas aquellas al derredor de la articulación internacional/global de espacios de regulación que (1) surgen en temas de los cuales desplazan y confrontan la regulación estatal o (2) se ocupan de temas nuevos, en ámbitos (espaciales y/o materiales) a los cuales la acción estatal no llegó. Como alerta Sol Picciotto (2002, 2003 y 2005), la regulación internacional de la OMC tiene importantes límites, sea porque la presuposición neoliberal de qué el libre comercio y las reglas que impiden los Estados de intervenir en los derechos individuales no es suficiente para cubrir temas necesarios, como medio ambiente y distribución internacional de la riqueza, sea porque el formalismo jurídico basado en el paradigma legalista no tiene los instrumentos suficientes para extender los ámbitos de legitimación para más allá de la atención a los intereses de los Miembros, o sea, los Estados, sin llegar a la sociedad civil o a los ciudadanos.

Esas tensiones están presentes en la formación de campos jurídicos internacionales, comprendidos, a diferencia de lo que Dezalay y Garth (1995 y 1996) describen como la creación del arbitraje internacional a partir de dobles imbricaciones con transferencias de capitales culturales y sociales de las esferas internas a la internacional y viceversa, como articulados en el entorno de esquemas burocráticos a los cuales los Estados atribuyeron competencias, o sea, en alguna medida y sentido son tomadas en préstamo del poder estatal. Sin embargo, la derivación descrita en la teoría jurídica no es suficiente para explicar el fenómeno de la jurificación internacional. 
Conforme la formulación estándar de los teóricos del Derecho Internacional Público, los órganos y Organizaciones Internacionales tienen personalidad y competencias como resultado único y exclusivo de la atribución específica en documentos internacionales, en especial los tratados constitutivos y, por lo tanto, son secundarios en su existencia y funciones y, además, precarios, puesto que dependen de la continuidad de la voluntad de los Estados para que sigan existiendo. En la práctica instituciones internacionales, aunque originalmente derivadas, pueden lograr obtener alguna autonomía social y política en relación a sus creadores, como se puede observar en la el Derecho supranacional europeo o en la acción efectivamente autónoma de organismos de las Naciones Unidas. La explicación de cómo eso se pasa, particularmente mediante la autonomización de campos jurídicos, va más allá del Derecho Internacional clásico y depende, también, de la compresión de la dinámica de relaciones interindividuales.

En ese sentido, no se trata, primariamente, de las relaciones entre Estados. Aunque se pueda hablar de Estados como actores sociales colectivos capaces de producir culturas, identidades y sentidos para sus acciones en relación a otros Estados e instituciones o organizaciones (WENDT, 1999), es muy difícil - y posiblemente inadecuado - articular una sociología de campo que los tenga por sujetos, aunque el propio Bourdieu lo hace, respeto a la economía internacional, considerando las corporaciones actores colectivos capaces de acciones estratégicas de interacción (2001 [1983] y 2001a). A diferencia de las corporaciones privadas en ámbitos económicos oligopolísticos, los actores sociales que articulan el campo jurídico - sobre todo jueces y académicos - son individuales ${ }^{1}$.

En ese artículo se propone que la OMC ha logrado un grado más amplio de autonomía en relación a sus Miembros a partir de las instancias de solución de disputas. La comprensión de la jurificación como la construcción de discursos y ambientes coherentes con el formalismo y el legalismo, aunque insuficiente para abarcar todos los ámbitos globales de legitimación, se ha mostrado eficiente para una bien sucedida autonomización del SSC de la OMC, ya sea como resultado inesperado de comprensiones sinceras de que los Miembros deseaban un órgano judicial de solución de disputas $^{2}$, ya de la elaboración de estrategias ${ }^{3}$ de diferenciación e independencia de las estructuras formales.

Las transformaciones de la composición de los grupos especiales y la estructura de distribución geográfica y basada en las experiencias profesionales en el OA ayudan a aclarar de que manera la independencia del control político por el OSD, combinada con la estructuración hecha por los primeros componentes del OA, han sido responsables por una autonomización del SSC en relación a los Miembros, mediada por la creación de un campo jurídico internacional con capacidad para inmunizar técnicamente las decisiones y contribuir para la legitimación del SSC y, con ello, de la OMC. 


\section{COMPONENTES DE los ÓRganos Des SSD DE LA OMC}

\section{I GRUPOS ESPECIALES}

La población de juzgadores de grupos especiales es formada por los nombres de componentes indicados en los instrumentos de constitución de los grupos especiales por el OSC ${ }^{4}$ desde el inicio de 1995 (vigor de la OMC) al final de 2008. Hay 136 accesibles, cada uno con tres nombres, totalizando 408 individuos, los cuales corresponden a 185 personas 5 .

Se trazaron los perfiles según los datos de los instrumentos indicados supra, cuales sean (1) presidente o componente simple y (2) procedimiento de indicación por las partes o por el Director-General. Además se realizó una búsqueda detallada en Internet con Google Web, Google Books, Google Academic, búsquedas de personas y publicaciones en www.lexisnexis.com (servicio académico), www.westlaw. $\operatorname{com}^{6}$ y búsquedas de libros en www.amazon.com y www.amazon.fr. Además de los nombres, se utilizaron como argumentos de búsqueda "biografía", "currículo", "derecho", "universidad", "oficina”, "bufete”, "ministerio", "gobierno”, "embajador", "secretario", “consejero", “doctor", "profesor", “OMC” y "Organización Mundial del Comercio", con sus correspondientes en francés e inglés, los otros idiomas oficiales de la OMC. Se consideraron para el computo en los perfiles datos de los websites de la OMC, estatales, de Organizaciones Internacionales oficiales, oficinas de abogacía y personales. En esa investigación los datos han servido para identificar si el individuo tiene: (1) formación jurídica; (2) carrera diplomática; (3) carrera académica y (4) otras funciones públicas, además de la nacionalidad de cada uno.

Aunque existen resultados interesantes en términos de la distribución de género y de la indicación de los componentes por el Director-General, eses no serán presentados o discutidos en ese artículo, que se concentra en los perfiles de formación y carrera. En ese sentido, la hipótesis de base de la jurificación, el triunfo de los abogados sobre los diplomáticos de Michael K. Young (1995), se reflejaría en una distribución en que la mayor parte de los individuos tenga formación jurídica y que los diplomáticos sean menos presentes en los grupos especiales. Los resultados, sin embargo, no corroboran con tanta fuerza esa percepción y, consecuentemente, exigen una explicación más detallada y circunstanciada, como se presentará en el tópico siguiente.

Por ahora, es importante ojear los datos. La distribución por carrera en el agregado del 1995 al 2008, calculada con admisión de carreras dobles, como, por ejemplo, la de un profesor que es, a la vez, abogado, la cual cuenta puntos para las dos categorías es la siguiente (Gráfico 1): 


\section{GrÁFICO I}

Distribución por carrera en GEs: Agregado 1995-2008

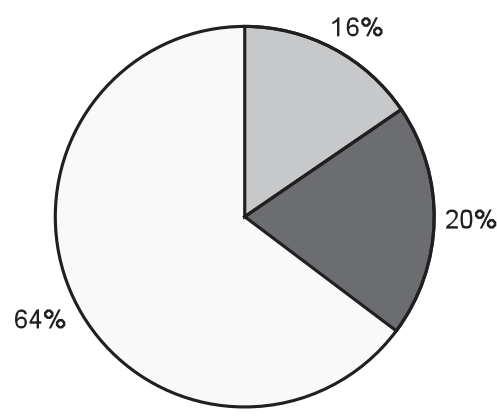

$\square$ profesionales
$\square$ profesores
$\square$ diplomáticos

La presencia de diplomáticos es claramente predominante, lo que apunta para un triunfo legalista, por lo menos en principio, bastante dudoso. Es cierto que la distribución cronológica de estos mismos datos podría aportar un color distinto. Una vez más, la hipótesis sería la de que profesionales y profesores ganarían mayores participaciones en relación a los diplomáticos, lo que efectivamente ocurre, como se observa abajo (Gráfico 2). Hubo, sin duda, una tendencia inicial de incremento de profesionales y profesores, que se estabilizan al derredor de participaciones de $20 \%$. Sin embargo, sería contrario a la razón decir que estos tienen, hoy por hoy, una participación que predomina sobre la de los diplomáticos, la cual se mantiene en un patatar entre $70 \%$ y $80 \%$, cerca del promedio histórico de 78\%. La proporción de diplomáticos, que ha sido la del $100 \%$ en 1995 , a penas ha bajado del $70 \%$ en 2004 , pero en los demás años se mantiene muy cerca de los $80 \%$.

\section{GRÁFICO 2}

\section{Distribución por carrera en GEs Variación por períodos}

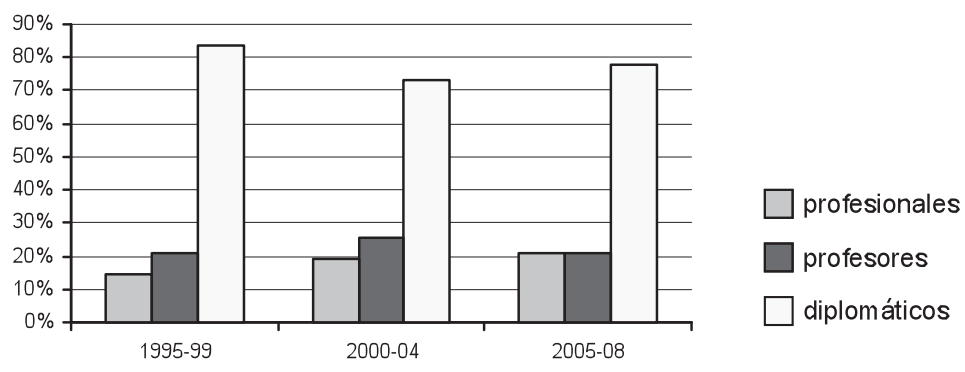


046 : SOLUCIÓN DE DISPUTAS EN LA OMC: UN ANÁLISIS A PARTIR DE LA SOCIOLOGÍA DE PIERRE BOURDIEU

Además de la presentación de los resultados cuanto a la distribución en términos profesionales, es importante evaluar la participación de los juzgadores con formación jurídica. En ese sentido, hay un crecimiento muy claro de la participación de estos componentes, ya en la función de presidentes, ya como simples miembros, como se puede observar abajo (Gráfico 3):

\section{Gráfico 3}

Formación jurídica en grupos especiales:

Presidentes y componentes simples

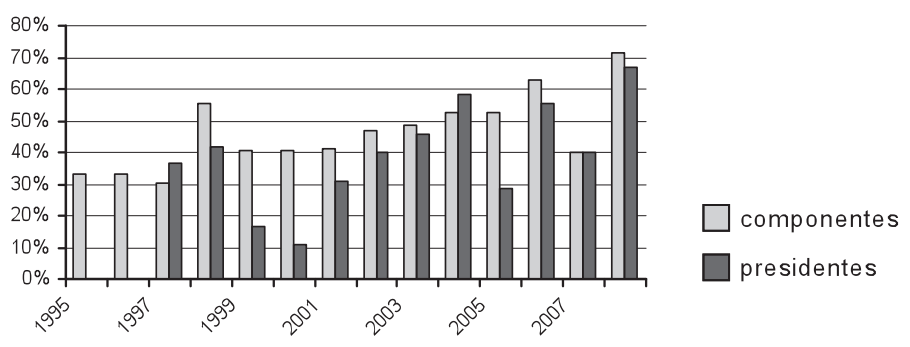

Los promedios agregados de componentes con formación jurídica, 46\%, y presidentes en la misma condición, 34\%, ha sido superadas en 2003, con a penas una excepción para cada una: 2007 para componentes y 2005 para presidentes; desde el 2003 al 2008 los promedios suben para 55\% y 49\%, respectivamente. Además, otra interesante transformación cronológica se pasa con los perfiles de los grupos, los cuales han, casi sin excepción, dejado de funcionar sin algún componente con formación jurídica desde el 2005, como se puede ver en el Gráfico 4:

\section{GRÁFICO 4}

\section{Grupos especiales sin componentes con} formación jurídica

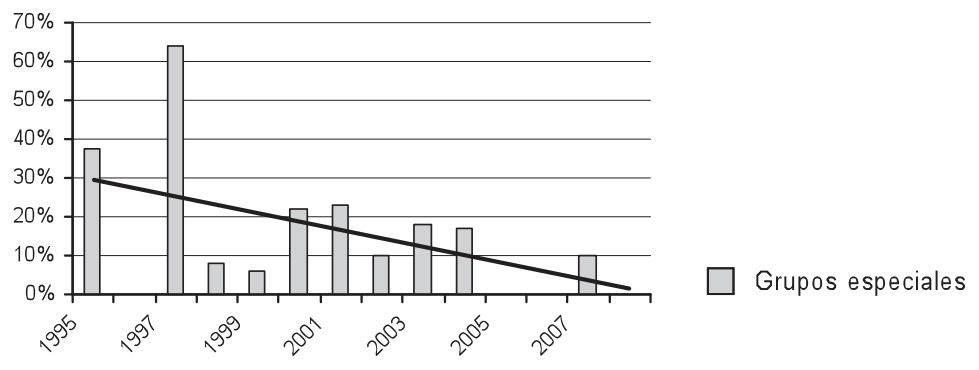


La evaluación de los cambios cronológicos indica una clara tendencia incremental de la participación de componentes con formación jurídica, sin que todavía sea clara la formación de una asíntota horizontal, lo que indica que tales participaciones, todavía al derredor del 50\%, tienen espacio para crecer. Sin embargo, el crecimiento de la participación de individuos con formación jurídica es superior al de los abogados y profesores. Como se observa en el Gráfico 5, es difícil afirmar que el incremento de los juzgadores con formación jurídica sea una función directa de la variación de los no diplomáticos, lo que es particularmente claro a partir del 2003. En otras palabras, por lo menos desde esa fecha, el incremento de la proporción de componentes de grupos especiales con formación jurídica se debe a la elección de más diplomáticos que pertenezcan, también, a esa categoría.

\section{GrÁFICO 5}

\section{Grupos especiales: no-diplomáticos y personal con formación jurídica}

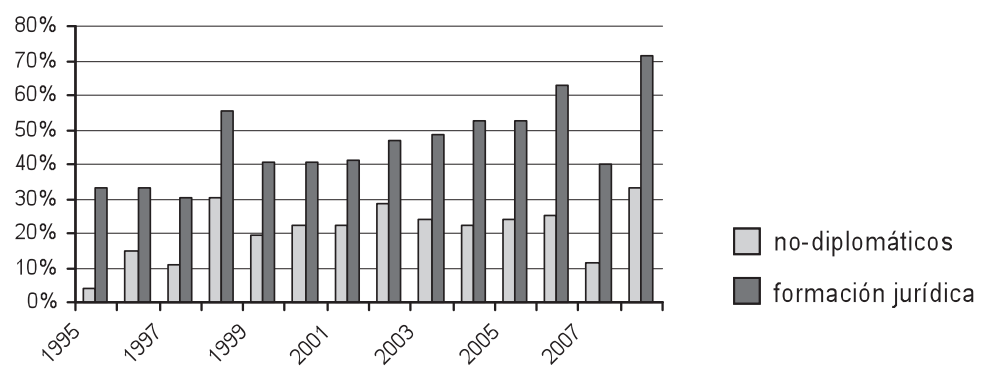

El Gráfico 6, con el agregado por carrera desde 2003 hasta 2008, es muy similar al agregado general: los diplomáticos pierden a penas un punto porcentual para los profesionales, mientras que el promedio de juzgadores con formación jurídica varía del $45 \%$ al 53\%, con un delta de ocho puntos porcentuales.

Por los datos presentados, es posible afirmar que la predominancia de los diplomáticos en los grupos especiales sigue siendo un rasgo muy claro de eses órganos de arreglo de disputas. Aunque la participación de personal con formación jurídica se presente con una tendencia de crecimiento, las causas de esto varían cronológicamente: en un primer período (1995 a 2001) los profesores superan ampliamente los profesionales, a excepción de 1997, pero luego (2002 a 2008) hay un incremento más consistente de los profesionales, el que sirve como impulso a los profesores, que no experimentan una caída significativa, aunque, en 2008, vuelven a tener una participación de más de 30\%. La tendencia de crecimiento del número de profesionales, que sugiere una estabilización al derredor del 20\% de los miembros de grupos especiales, 
es más clara que la de los profesores, qué,luego de un crecimiento hasta el 2003, sufren una caída consistente, con la excepción de 2006 y 2008, lo que apunta, por lo menos, para una variación más errática.

\section{GrÁFICO 6}

Destribución cronologia (1995-2007):

Formación jurídica, profesores y profesionales

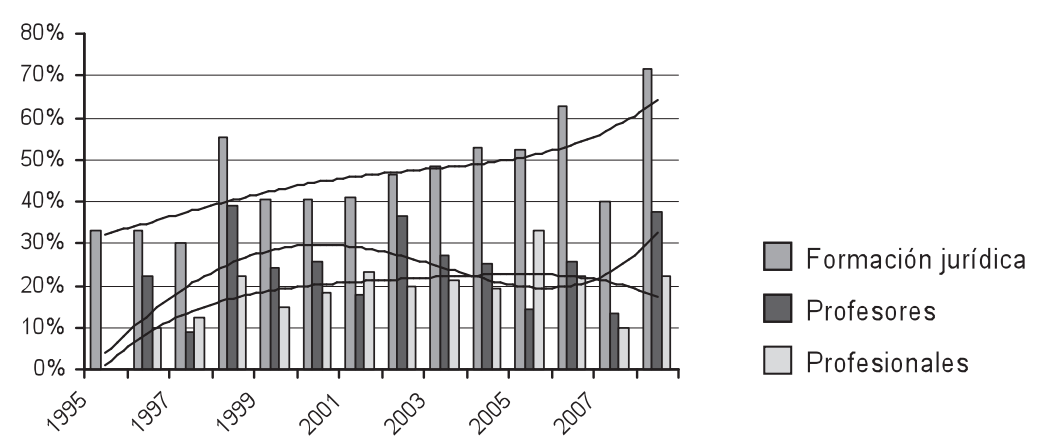

Las dos tendencias más marcadas, por lo tanto, son las de (1) crecimiento del número de juzgadores con formación en Derecho y (2) de presencia predominante de personal vinculado a la diplomacia y funciones públicas. Las variaciones en términos de participación de profesores y profesionales tiene la apariencia de resultado de una búsqueda, por tentativa y error, de ajustar la actuación y los resultados de los grupos especiales. En ese sentido, las transformaciones de distribución de perfiles sugieren adaptaciones reactivas para incrementar niveles de éxito de los grupos especiales.

\section{2 Órgano de Apelación}

En el sitio de Internet del OA hay información detallada respeto a sus miembros, con breves biografías que abarcan no a penas su carrera anterior, pero las actividades que ejercen luego de su participación en el órgano. Se utilizaron eses datos para atribuir los calificativos de sus componentes, sin profundizar la búsqueda con otros criterios, porque (1) eses representan la imagen que se quiere proyectar en el ámbito del OA y (2) se supone que siguen todos los mismos criterios y procesos de composición. Sin embargo, alguna investigación adicional se hizo respeto a las oficinas de abogacía en las cuales algunos de los miembros ejercen su actividad, utilizándose los respectivos sitios de Internet.

El OA es permanente y, por lo tanto, no se necesita de la diferenciación entre individuos y personas que se hizo en el tópico anterior. La población es formada por componente/año, o sea, la persona cuenta como un integrante de esa para cada año 
en que mantiene - por todo el periodo o parcialmente - vínculo formal con el OA. Los agregados y promedios no se calculan con basis en las personas, pero tienen en cuenta cada composición anual del órgano. El periodo cubierto va de 1995 hasta 2009. Para ese último año, se incluye la conformación que se supone que será la de fines de diciembre.

En lo que se refiere a las carreras, se hizo un esfuerzo para identificar la principal carrera del componente (profesional, profesor o diplomático) a partir de dos criterios: (1) el cronológico, según el cual la principal carrera es la última que antecede la jubilación y (2) sobre todo entre los que son, a la vez, profesores y abogados, una ponderación que consideró como predominantemente abogados los que trabajan en oficinas internacionales y, asimismo, no se consideró como profesores aquellos que no tienen una vinculación formal continua con una universidad. Ha sido posible, por lo tanto, conformar dos matrices: la A, que refleja los dobles y triples perfiles, y la $\mathrm{B}$, para las carreras predominantes.

El agregado de la matriz A indica como perfil típico el de un hombre (89\%) de 65 años, que estudió Derecho (88\%) y tuvo alguna función diplomática o de carrera pública asociada al comercio exterior (69\%), ejerció función de profesor universitario (75\%) y estudió en el exterior (65\%). Del agregado, a penas 40\% actuaron como abogados y 6\% como juez. La composición de fines de 2009 es, sin embargo, distinta: 57\% de varones, promedio de edad de 60 años, 85\% de diplomáticos, $85 \%$ de profesores, $57 \%$ de profesionales jurídicos. De todos, a penas Shotaro Oshima, diplomático japonés, todos los demás tienen dos o tres carreras en el perfil de la matriz A.

Al observarse la representación de las proporciones del agregado histórico, en el Gráfico 7, o la de julio de 2009, en el Gráfico 8, queda claro que la composición presente no es muy distinta a la del agregado, aunque en comparación con el Grafico 1 se presentan diferencias muy sensibles. Aunque la presencia de individuos con carreras diplomáticas sea todavía muy importante en el órgano de apelación, a la diferencia de los grupos especiales esta no es claramente predominante y la presencia de profesores entre los componentes del OA es, desde su primera formación, por lo menos tan importante cuanto la de diplomáticos. En ese sentido, el OA es más parecido a otras cortes internacionales, como la Corte Internacional de Justicia y las cortes de Derechos Humanos, en las cuales las credenciales académicas son, en general, evaluadas como activos importantes (TERRIS et al.: 2007). 
050 : SOLUCIÓN DE DISPUTAS EN LA OMC: UN ANÁLISIS A PARTIR DE LA SOCIOLOGÍA DE PIERRE BOURDIEU

\section{GRÁFICO 7}

Distribución por carrera en el OA:

Agregado 1995-2009 (matriz A)

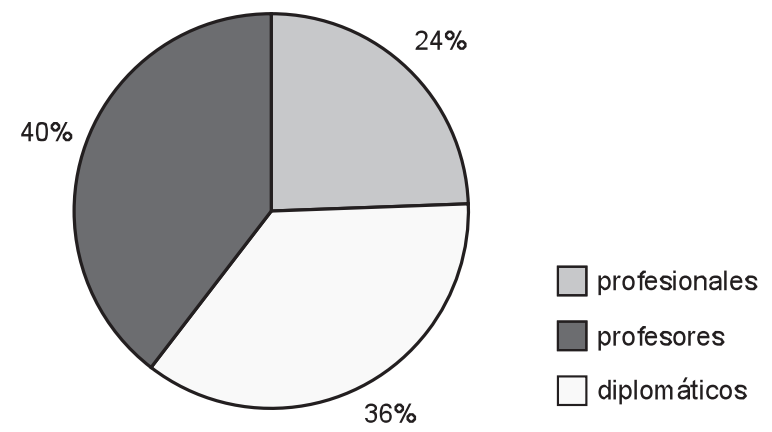

\section{GrÁFICO 8}

Distribución por carrera en el $0 A$ :

Julio de 2009 (matriz A)

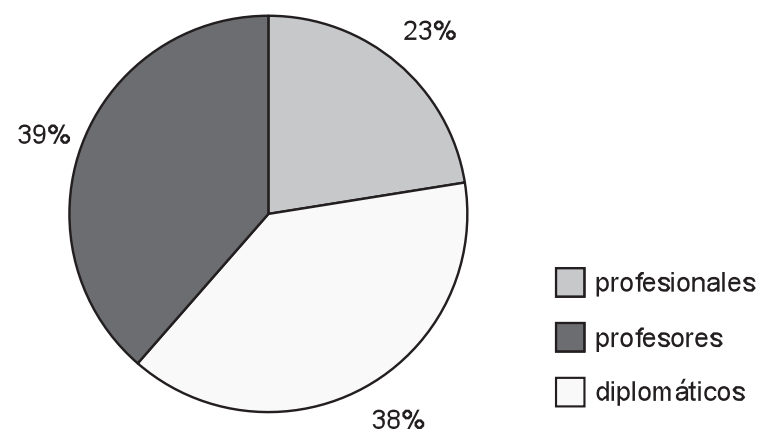

Las variaciones en términos de edad de los componentes, por su turno, presentan una tendencia de disminución, aunque los mandatos de cuatro años y las muy frecuentes recondiciones de sus componentes suelen producir períodos de crecimiento constante a la razón de un año agregado al promedio a cada año, como se observa en la primera parte (1995 hasta 1999) y en el periodo del 2003 al 2005 del Gráfico 10 . 


\section{GRÁfICO 9}

Promedio de la edad en el OA: 1995 hasta 2009

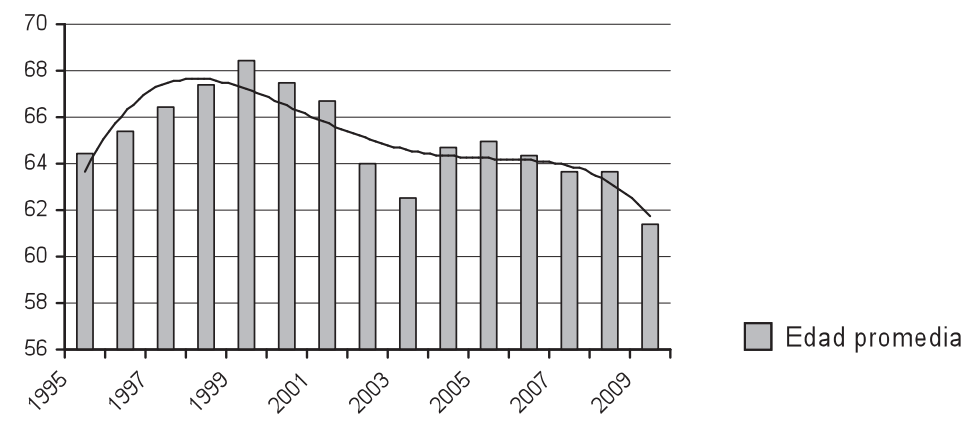

Teniendo en cuenta las distribuciones por edad cruzadas con las carreras de la matriz B (predominantes), se puede atribuir el cambio en los promedios de edad a la indicación de diplomáticos más jóvenes. En el Grafico 11 se puede observar que la variación por edad agregada de los profesores y profesionales ${ }^{7}$ es pequeña, aunque suavemente descendente, pero la de los diplomáticos disminuye a una tasa promedia de 2 años por quinquenio.

\section{Grafico io}

Variación de edad por quinquenio en el $O A$ : diplomáticos y profesores + profesionales

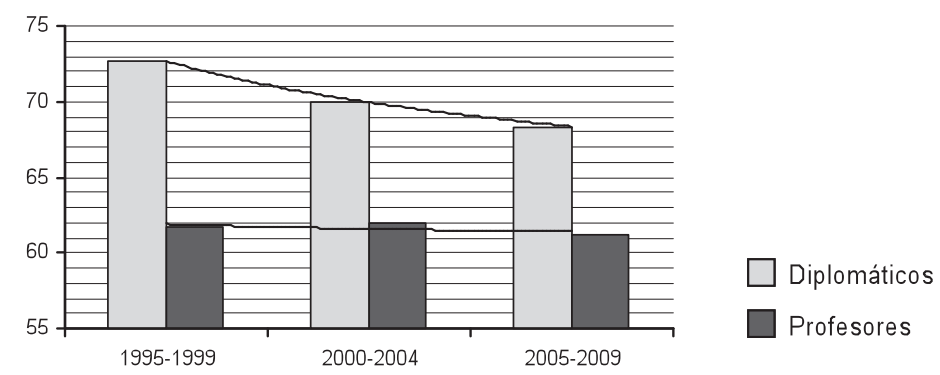

La variación general por edad se explica, por lo tanto, con referencia al menor promedio en el ingreso de los diplomáticos. Eso sugiere algunas posibles explicaciones causales relacionadas con la percepción original del órgano y sus modificaciones, las cuales, sin embargo, serán presentadas más adelante. 
Por el momento, es importante subrayar algunos aspectos importantes respeto a la composición del OA. En primer lugar, aunque la presencial de diplomáticos sea mucho más amplia en ese órgano que en las cortes y tribunales internacionales, es hecho que su proporción es muchísimo menor que en el promedio de los grupos especiales, incluso si teniéndose en cuenta su período más reciente. La proporción de profesores es bastante elevada, lo que es consistente con el interés en generar legitimidad a partir de habitus propios de la composición de los campos jurídicos (BOURDIEU: 2001b), presente en las cortes internacionales de Derecho Internacional Público (TERRIS et al.: 2007) y en los tribunales arbitrales comerciales y entre inversionistas y Estados (DEZALAY; GARTH: 1995; 1996; COSTA: 2009). La conformación del OA en términos de carreras anteriores es, por lo tanto, consistente con la inmunización académica para, asociada al legalismo y a la presunción de imparcialidad, construir espacios de imposición de soluciones basadas en el Derecho, las cuales son, aquí, estrictamente comprendidas como las que no revelan las relaciones de poder subyacentes, las cuales se inmunizan por la actuación de órganos que construyen su influencia a partir de la generalización de la aceptación social de su carácter justo y preferencial.

\section{Conclusiones}

El OA surgió como un substituto al control por las Partes Contratantes, reemplazada por el OSD en la OMC. Con la adopción de una sistemática de aprobación automática de los informes de grupos especiales si no se produce consenso en contra, los Miembros sintieron necesidad de crear un órgano de control cuya efectiva utilización, se esperaba, sería excepcional (HUDEC: 1999; HUGHES: 2009). En la elaboración normativa de los procedimientos hecha en el Entendimiento sobre Solución de Disputas (ESD), los grupos especiales siguieran las líneas generales ya establecidas en la historia del GATT, pero no había un precedente para el OA y los negociadores dejaron a sus componentes la competencia de regularlos, lo que se hizo en los Procedimientos de Trabajo del Órgano de Apelación (PTOA).

La posición de los primeros componentes del OA ha sido la de poner en funcionamiento un sistema para favorecer la juridicidad de las decisiones y, asimismo, la legitimidad en faz de los Miembros, sin olvidarse de posibles otros auditorios. La opción por una collegiality fuerte, con las divisiones, formadas por tres componentes, encargadas de decidir las cuestiones presentadas en apelación de los informes de grupos especiales y, además, someter a los otro cuatro componentes para una discusión con el objetivo de impedir que se tomen decisiones contradictorias en casos semejantes, lo que se ha mostrado un sistema muy eficiente (ÁLVAREZ-JIMÉNEZ: 2009). No se puede olvidar que el fuerte compromiso de no emitir opiniones divergentes ha sido ampliamente respetado y, hasta el presente, a penas se han presentado en dos ocasiones ${ }^{8}$. 
Además, la utilización del OA se hizo mucho mas significativa de lo que se imaginaba originalmente. En los dos primeros años de funcionamiento del órgano, todos los informes de grupos especiales han sido apelados y, en los 15 años de su actuación, el promedio agregado es del 68\%. Es muy normal, por lo tanto, que los informes de grupos especiales sean apelados y esa situación presiona para que los grupos especiales - o las instancias burocráticas de su nombramiento (el Secretariado y el Director-General) - a la adecuación qué, como se observó muy claramente en los datos levantados, significó el incremento de la proporción de personal con formación jurídica.

Los datos referentes a las composiciones de los grupos especiales son claros en indicar una tendencia de crecimiento de la participación de personal con formación jurídica en los procesos de tomada de decisión. A la primera mirada eso apunta hacia un fortalecimiento del carácter específicamente jurídico del SSD de la OMC, aunque en los grupos especiales el promedio de diplomáticos en el agregado de los 15 años de funcionamiento considerados no es diferente del de los años mas recientes y parezca haberse estabilizado al derredor del $80 \%$, aunque entre estos se haya hecho más frecuente el nombramiento de aquellos con formación jurídica.

La composición del OA, por su turno, se muestra bastante estable en términos de perfiles profesionales y no ha variado significativamente en los casi 16 años evaluados. La participación combinada de diplomáticos y profesionales de 75\% (matriz A) o más (matriz B) es importante para la construcción de la legitimidad del órgano, que se apoya, a la vez, en la reputación técnica de los académicos y en la experiencia y percepción política de los diplomáticos. Aunque en minoría, los profesionales refuerzan la legitimidad técnica y aportan la vivencia específica en campos jurídicos internos. Ese equilibrio entre visiones políticas y técnicas podría, en tesis, llevar a una relativización del formalismo jurídico en relación a las percepciones políticas, pero no es eso lo que se verifica.

Por lo tanto, las estrategias de adaptación de los grupos especiales se producen en faz de las siguientes constataciones: (1) ha dejado de ser fundamental el convencimiento de las partes y, por consiguiente, el cumplimiento voluntario sin que se bloqueen los informes en las PARTES CONTRATANTES/OSD; (2) si no es posible convencer las partes, es importante convencer al OA, para que ese no reformule los informes y, sobre todo, sus resultados; (3) para convencer las partes es necesario negociar y buscar soluciones equilibradas, en las cuales no es necesario que haya la aplicación estricta de la ley, pero para convencer a los órganos de apelación el discurso legalista es más efectivo, lo que ha pasado, también, con los grupos especiales y (4) la incorporación de personal jurídico - hoy son muy raros los grupos especiales sin ellos - sirve para adecuar la técnica legalista a las decisiones. Tales constataciones llevan a estrategias específicas, las cuales se verifican en el análisis de la composición de grupos especiales, pero tienen como efecto colateral la generación 
de un campo jurídico, el cual se caracteriza por la legitimación legalista y, en el caso, por la importación para el ámbito internacional de los habitus y capitales sociales propios de los campos nacionales.

Por su turno, el OA ha adoptado, desde el principio, una postura legalista y formalista. Sol Picciotto (2005) describe tal posición a partir de la adopción de una epistemología objectivista y un estilo formalista, los cuales derivan de la estructura institucional que limita procesos de legitimación a los auditorios estatales y, por lo tanto, a la opción por una acción formalista. Sin embargo, es importante resaltar que esa interpretación no permite atribuir al órgano una actitud pasiva o neutral: sobre todo la primera composición histórica del OA tuvo una postura muy activa para su afirmación como órgano (1) técnico y no político y (2) con una estructura interna fuerte, basada en una collegiality de dos niveles y en el compromiso consciente en no crear manifestaciones divergentes.

Si los grupos especiales tienen por auditorios relevantes (1) las partes en las controversias y (2) el OA, ese órgano, preocupado con su fortalecimiento y su autonomización respeto al OSD, identifica como principales plateas (1) los Miembros de la OMC y (2) instancias internacionales no estatales capaces de influenciar las políticas internas de los Estados y de actuar globalmente, ya de la sociedad civil organizada, ya del sector empresarial. La estrategia legalista, pero con aberturas para intereses distintos de los estatales, vienen marcando la actuación del OA en eses años de funcionamiento. La composición estable y el suceso en frente a los Miembros puede servir para estabilizar la sistemática vigente $\mathrm{y}$, por consiguiente, favorecer el establecimiento de un campo jurídico de la OMC que será muy similar a los internos.

: ARTIGO APROVADO (10/07/2010) : RECEBIDO EM 27/01/2010

NOTAS

1 Es posible abarcar actores colectivos en algunas posiciones, como las grandes firmas internacionales o cortes internacionales con fuerte carácter colegiado, como el OA de la OMC.

2 Por ejemplo, Júlio Lacarte-Muró (2007, p. 40), diplomático uruguayo con larga experiencia en el GATT y componente del primer grupo de miembros del OA, afirma: "Y cuanto al OA? (...) A penas puede haber una conclusión. El OA existe para aplicar imparcialmente las normas de la OMC, como establecido por los Miembros. El OA es el guardián de la legalidad de la OMC u no hay espacio para tener en cuente las reacciones o voluntades políticas, sean cuales sean.” 
3 Por ejemplo, Georges Abi-Saab (2006, p. 4), componente del OA de 2000 a 2008, afirma qué "él OA, desde el principio, consciente y sistemáticamente ha afirmado y consolidado su carácter judicial, tanto en las formas de funcionamiento, cuanto en el proceso de razonamiento."

4 Disponible en www.wto.org/english/tratop_e/dispu_e/find_dispu_documents_e.htm, consultado entre 10 y 14 de abril del 2009.

5 Acá, individuo es una ocurrencia de indicación de nombre. Una persona es un agregado de individuos con nombres idénticos.

6 El acceso a la Westlaw ha sido proporcionado por la Universidad Torcuato di Tella (Buenos Aires), de 16 a 30 de junio de 2009, durante corta estada de investigación.

7 La población de profesionales predominantes (matriz B), de la cual representa $8 \%$ y su distribución demasiado errática. Como los profesionales en cuestión también se presentan como profesores, se eligió agregar las dos categorías, lo que, además, tuvo poco impacto en el agregado de los profesores, igualmente estable y en el mismo nivel.

8 EC-Asbestos y US - Upland Cotton.

\section{REFERÊNCIAS BIBLIOGRÁFICAS}

ABBOTT, Kenneth W.; KEOHANE, Robert O.; MORAVCSIK, Andrew; SLAUGHTER, Anne Marie; SNIDAL, Duncan. "The concept of legalization". In: International Organization. v. 54. n. 3. Cambridge, MA: IO Foundation and the MIT, summer 2000.

ABI-SAAB, Georges. “The appellate body and treaty interpretation”. In: BOHANES, Jan; SACERDOTU, Giorgio; YANOVICH, Alan. The WTO at ten: the contribution of the dispute settlement system. Cambridge: Cambridge University Press, 2006. Disponible en http://www.abanet.org/intlaw/spring06/materials/Abi-

Saab.WTO.TreatyInterpret.pdf, consultado 12 de abril de 2009.

ÁLVAREZ-JIMÉNEZ, Alberto. “The WTO Appellate Body decision-making process: a perfect model for international adjudication?” In: Journal of International Economic Law. v. 12. n. 2. Oxford: Oxford University Press, 2009.

BOURDIEU, Pierre. Las estructuras sociales de la economía. Buenos Aires: Manantial, 2001a. O poder simbólico. Rio de Janeiro: Bertrand Brasil, 2001b. $4^{\mathrm{a}}$ Ed.

. The forms of capital. [1983] . In SWEDBERG and GRANOVETTER (Orgs.), The Sociology of Economic Life. Boulder: Westview, 2001. $2^{\mathrm{a}}$ Ed.

CARTLAND, Michael. "Comment on a WTO permanent panel body". In: Journal of International Economic Law. v. 6. n. 1. Oxford: Oxford University Press, 2003.

COSTA. José A. F. “A imparcialidade na arbitragem entre investidores e Estados”. In: Revista de Mediação e Arbitragem. n. 21. São Paulo: RT, 2009.

DEZALAY, Yves. "Les courtiers de l'international: héritiers cosmopolites, mercenaires de l'impérialisme et missionnaires de l'universel”. In: Actes de la Recherche en Sciences Sociales. n. 151-152. Paris: Seuil, 2004.

DEZALAY, Yves; GARTH, Bryant. "Setting the legal scene for North-South disputes and te collective construction of the universality of law". In: American Bar Foundation Working Paper Series. n. 9.405. Chicago: American Bar Foundation, 1995.

. Dealing in virtue: international commercial arbitration and the construction of a transnational legal order.

Chicago: Chicago University Press, 1996.

HUDEC, Robert E. "The new WTO dispute settlement procedure: an overview of the first three years". In:

Minnesota Journal of Global Trade. n. 8. v. 1. 1999.

HUGHES, Valerie. “The institutional dimension”. In: BETHELEHEM, Daniel; McRAE, Donald; NEUFELD, Rodney; VAN DAMME, Isabelle (Orgs.). The Oxford handbook of International Trade Law. Oxford. Oxford: Oxford University Press, 2009.

JACKSON, John H. The world trading system: law and policy of international economic relations. Cambridge (MA): MIT Press, 1997. 
LACARTE-MURÓ, Julio A. "Os primeiros anos do Órgão de Apelação e do sistema de solução de controvérsias na OMC: uma perspectiva histórica”. In: BAPTISTA, Luiz O.; CELLI JÚNIOR, Umberto; YANOVICH, Alan. 10 anos de OMC: uma análise do sistema de solução de controvérsias e perspectivas. São Paulo: Aduaneiras, 2007.

PICCIOTTO, Sol. Defending the public interest in Trips and the WTO. In: Peter DRAHOS, Peter; MAYNE, Ruth (Orgs.). Global intellectual property rights: knowledge, access and development. Londres: Palgrave e Oxfam, 2002.

. Private rights vs. public interest in WTO. In: Review of International Political Economy, v. 10, n. 3. 2003.

The WTO's Appelate Body: legal formalism as a legitimation of global governance. In School of Public Policy Working Paper Series, n. 14. London: University Collegy, 2005.

TERRIS, Daniel; ROMANO, Cesare P. R.; SWIGART, Leigh. The internacional judge: an introduction to the men and women who decide the world's cases. Watham: Brandeis University Press, 2007.

WENDT, Alexander. Social theory of international politics. Cambridge: Cambridge University Press, 1999. YOUNG, Michael K. "Dispute resolution in the Uruguay Round: lawyers triumph over diplomats". In: The international lawyer. n. 29. 1995.

Av. Brig. Luís Antônio, 3030 - Apto. 405 Jardim Paulista - 01402-000 São Paulo - SP - Brasil jafontouracostalagmail.com

\section{José Augusto Fontoura Costa}

DOUTOR EM DIREITO INTERNACIONAL PELA Universidade de São PAUlo

Professor Titular da Universidade de São Paulo (FDUSP), da Universidade Católica de Santos (UniSantos) E dA Universidade do Estado do Amazonas (UEA) 